\title{
Transanal Endoscopic Microsurgery: Endoscopy Assisted Treatment of Colorectal Anastomotic Stenosis
}

\author{
Giancarlo D’Ambrosio ${ }^{1}$, Antonietta Lamazza ${ }^{2}$, Rossella Palma $^{3}$, Andrea Picchetto ${ }^{1}$, Cristina Panetta ${ }^{3}$, \\ Antonello Trecca ${ }^{4}$, Stefano Pontone ${ }^{3}$, Emanuele Lezoche ${ }^{1}$ \\ ${ }^{1}$ Department of General Surgery, Surgical Specialties and Organ Transplantation "Paride Stefanini," Sapienza University, Rome; ${ }^{2}$ Department \\ of Surgery, Istituto Pietro Valdoni, Sapienza University of Rome, Rome; ${ }^{3}$ Department of Surgical Sciences, Sapienza University of Rome, Rome; \\ ${ }^{4}$ Department of Operative Endoscopy, USI Group, Rome, Italy
}

Transanal endoscopic microsurgery (TEM) is a type of natural orifice transluminal endoscopic surgery, developed for rectal tumors and used also to treat other rectal diseases. Anastomotic complications after colorectal surgery, including stenosis, represent a challenging problem. We present the case of a 36-year-old woman with a diagnosis of Hirschsprung disease that was submitted to a modified Duhamel operation. A postoperative barium enema showed a complete stricture of the anastomosis that was impossible to resolve by flexible endoscopic approach. Then an intraoperative endoscopic approach to facilitate the localization of preanastomotic colon (proximal colon from the anastomosis) was performed by a small colotomy and the colonic recanalization was obtained by the creation of a neo-anastomosis by TEM, under fluoroscopic-endoscopic control. The patient underwent a control barium enema showing regular retrograde transit of contrast medium without evidence of stenosis. In our experience, transanal approach by TEM-colonoscopy assisted is safe and feasible and represents a model of combined minimally invasive technique.

Keywords: Transanal endoscopic microsurgery; Anastomotic stenosis; Endoscopy; Hirschsprung disease; Duhamel

\section{INTRODUCTION}

Since its introduction in 2004, natural orifice transluminal endoscopic surgery (NOTES), has attracted a great deal of interest from surgeons and endoscopists [1]. Transanal endoscopic microsurgery (TEM), a type of NOTES originally developed in 1983 by Buess, is a minimally invasive transanal procedure used to excise benign and selected malignant tumors of the rectum. The main advantage offered by TEM is the use of an endoluminal approach, which eliminates the need of a transabdominal incision, improving hospital stay and quality of life. According to its tech-

Received: Apr 7, 2018 - Revised: Feb 16, 2019 - Accepted: Sep 30, 2019 Correspondence to: Rossella Palma, M.D.

Department of Surgical Sciences, Sapienza University of Rome, V.le Regina Elena n. 324 00161, Rome, Italy

Tel: +39-3343091174, Fax: 39-0649972447

E-mail: rossellapalma89@gmail.com

ORCID: https://orcid.org/0000-0002-1533-552X

(C) 2020 The Korean Society of Coloproctology

This is an open-access article distributed under the terms of the Creative Commons Attribution NonCommercial License (https://creativecommons.org/licenses/by-nc/4.0) which permits unrestricted noncommercial use, distribution, and reproduction in any medium, provided the original work is properly cited. nical characteristics, in particular magnification and brightness of the image, TEM is currently used also to treat other several diseases such as recto-vaginal and recto-urinary fistulas in order to avoid any incision in the perineal area and to preserve the sphincter functions [2]. TEM is a useful approach also to repair rectal perforation during colonoscopy and some cases of rectal wall endometriosis treated by TEM are also described. Anastomotic complications after colorectal surgery represent a challenging problem [3]. Benign stricture is a relatively common complication of colorectal anastomosis. Patients with symptomatic anastomotic stenosis typically complain about abdominal pain and cramps, constipation, and fractionated bowel evacuation. Symptoms may appear a few weeks to several years after surgery. In the majority of patients, an anastomotic stricture is a serious condition that may require repeated endoscopic treatment or surgery. Although this complication can be treated effectively by surgery, this is associated with significant technical difficulties, surgical risks and the possibility of recurrence. For this reason, the endoscopic approach is the first treatment option. A variety of endoscopic techniques have been described, but there is a lack of data from controlled prospective trials as to the optimal approach. Endoscopic 
dilation has become increasingly widespread and has proven to be a valid and safe treatment [4]. In patients with simple anastomotic obstruction, in whom balloon or boogie dilatation has failed, endoscopic stenting can represent a valid choice.

Some cases reported in the literature describe different endoscopic approaches, including endoscopic electrocision with a papillotomy knife or hook knife with or without balloon dilatation. A case of colorectal anastomosis web occlusion treated without electrocision in order to avoid thermal injuries has been also reported, using a suprapapillary biliary puncture catheter followed by balloon dilation [5]. Occasionally the anastomotic stenosis can be complete. In these cases, the endoscopic approach is technically difficult to perform, then surgery is required. Benign stricture is a reported complication also for the conventional surgery of Hirschsprung disease (HD). In order to reduce complication after surgery, various surgical techniques for HD have been developed and modified. Medical or surgical treatment should be adjusted based on strictures severity. We describe a rare case of postoperative complication of modified Duhamel operation consisting in a complete anastomotic stenosis, without orifice, treated by an innovative TEM-endoscopy assisted approach.

\section{CASE REPORT}

We present the case of a 36-year-old woman with intermittent abdominal distension, pain and constipation. HD was suspected by clinical symptoms, abdominal plain X-ray, and finally diagnosed by rectal biopsy. The patient was candidate for a modified $\mathrm{Du}$ hamel operation and a protective loop ileostomy was performed. Six months later, postoperative barium enema examination showed a complete stricture of the colorectal anastomosis without contrast opacification of the proximal tract (Fig. 1A). It was impossible to treat the stricture by flexible endoscopic approach, because no orifice could be detected by the endoscopist and the passage of the guidewire above the anastomosis wasn't possible (Fig. $2 \mathrm{~A})$. Then an intraoperative endoscopic approach to facilitate the visualization of preanastomotic colon was performed after we have received informed consent from patient. First of all, a small colotomy was performed and the patient underwent the intraoperative enteroscopy by using a $15 \mathrm{~mm}$ laparoscopy trocar with a sterile camera drape that was adopted to introduce a standard colonoscope through the colotomy. An adapted rectoscope for TEM was introduced from the anus and the colonoscope in the proximal tract of the intestinal lumen was visualized by transillumination (Fig. 3A, B). At this level an incision was done and a new endoluminal colorectal anastomosis was created by TEM under combined fluoroscopic-endoscopic control (Fig. 3C). Postoperative progress was regular and the patient was discharged in 7 th postoperative day (POD). The patient underwent a control barium enema on 30th POD and a regular retrograde transit of contrast medium was detected (Fig. 1B). The endoscopy showed an intact anastomosis without evidence of stenosis (Fig. 2B). Then the patient underwent a surgical operation for the closure of the ileostomy with regular restoration of intestinal canalization. An endoscopic 1-year follow-up didn't show stenosis recurrence and no other treatment was needed.

\section{DISCUSSION}

Benign anastomotic strictures can be amenable to balloon dilatation, with anatomical and clinical success in over $90 \%$ of cases at the time of intervention. However, in the medium term, the pro-
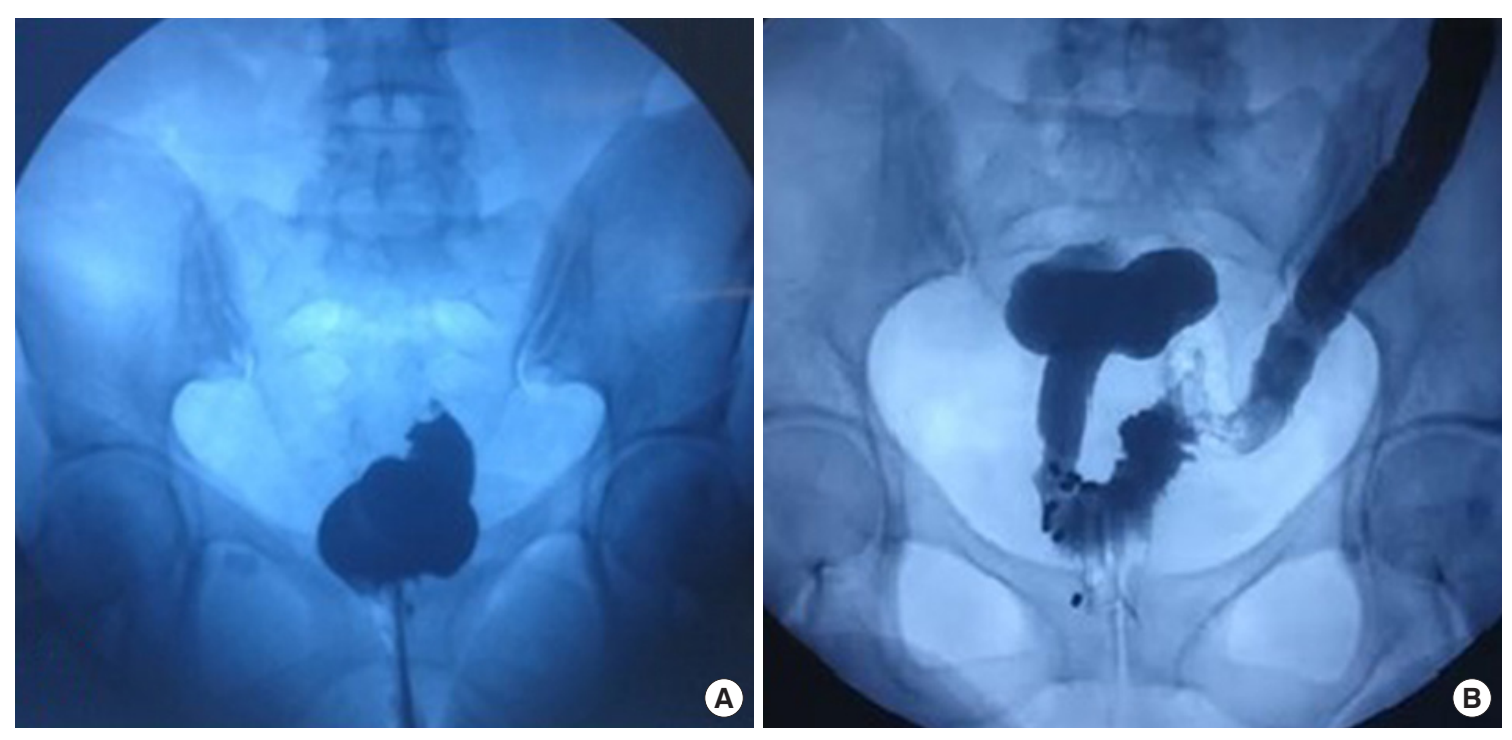

Fig. 1. (A) Preoperative contrast enema showing complete stricture of colorectal anastomosis. (B) Postoperative enema showing regular retrograde opacification of the preanastomotic intestinal tract. 

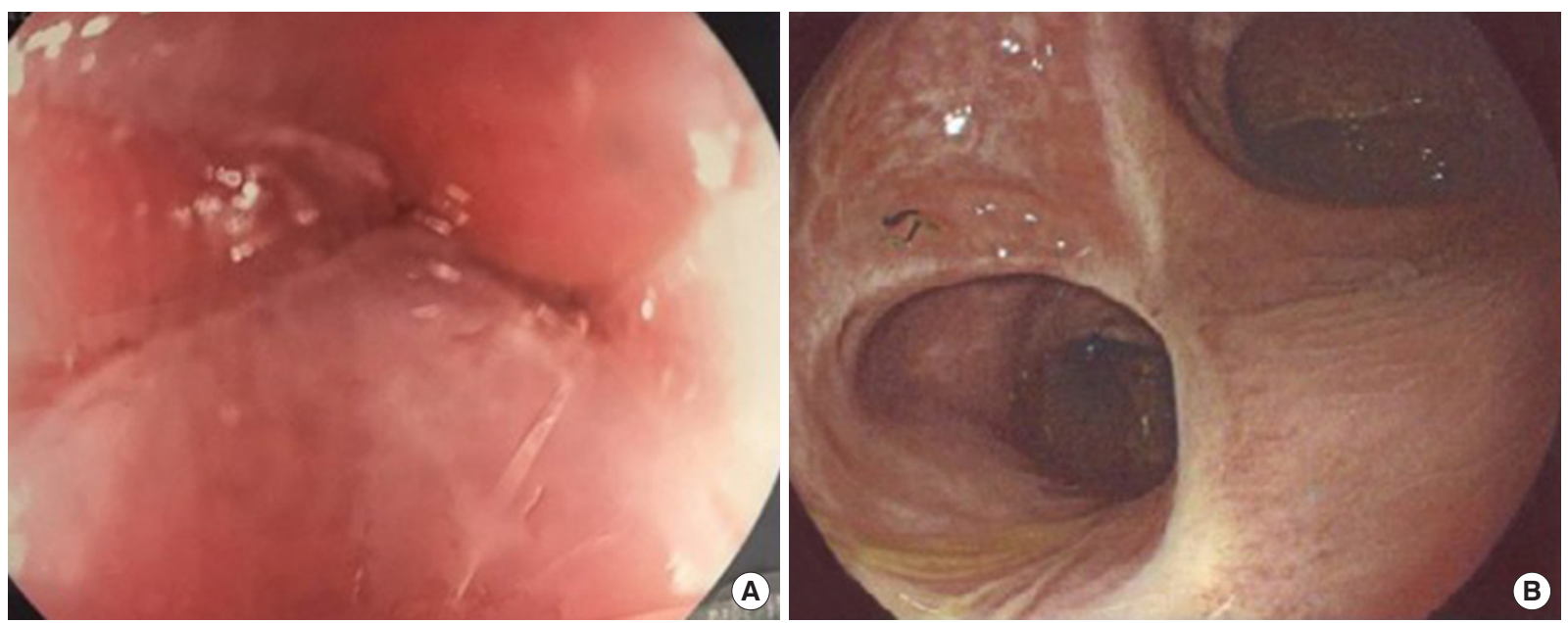

Fig. 2. (A) Preoperative endoscopy showing complete anastomotic stenosis. (B) Postoperative colonoscopy showing a regular and wide anastomosis.
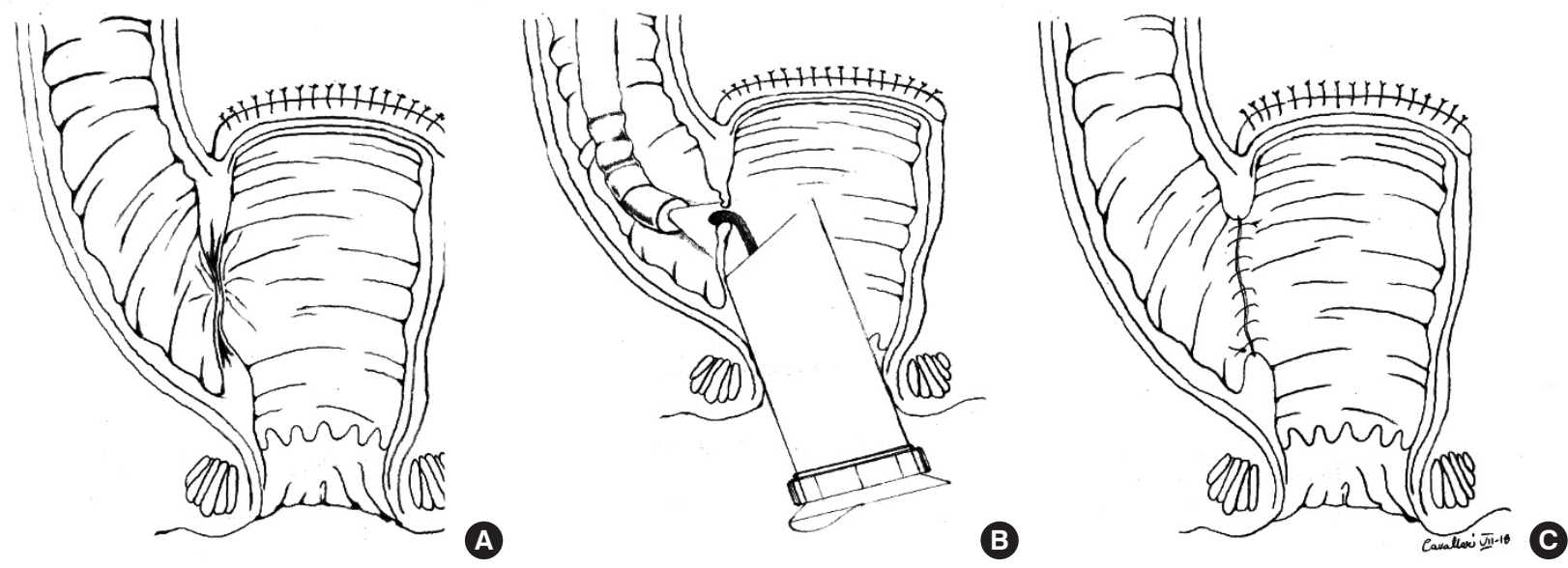

Fig. 3. (A) Schematic representation of complete anastomotic stricture. (B) View of the proximal colon by transilumination with a combined transanal endoscopic microsurgery-endoscopy assisted technique. (C) New colorectal anastomosis.

cedure is successful in only two-thirds of cases and recurrence of the obstruction is common and multiple dilatations are generally needed. Data from the literature about recurrence rates after endoscopic balloon dilation are scarce but have been reported between $6 \%$ and $20 \%$ with a follow-up of 2 and 1 year, respectively. Symptomatic anastomotic stenosis that do not respond to balloon dilation are usually resected but surgery is associated with a considerable morbidity and mortality. In the literature, major complications were reported in up to $18 \%$ of surgical procedures performed for anastomotic strictures [4]. Stents have been not so commonly implanted in patients with benign obstructive colorectal stenosis. Uncovered stents, which are very difficult to remove, are placed with reluctance in patients with a theoretical long life expectancy, like those with benign colonic obstruction. On the other hand, covered stents, which can be easily removed, migrate and dislodge with a high frequency with the possibility that the obstruction will recur.

Conventional surgery of HD is followed by early and late complications. In order to decrease these complications various surgical techniques have been developed and modified. It is generally believed that all postoperative complications, regardless of the original operative procedure, improved with time. A small number of patients have persistent stooling problems, often manifesting with either constipation or recurrent enterocolitis. Stricture formation has been proposed to be caused from different factors such as lack of blood supply, tension on the anastomosis, surgical technique, and postoperative management. Mild strictures can be corrected by medical treatments while reoperative surgery for $\mathrm{HD}$ typically falls into 2 categories: those who require a myectomy and those who need a redo pull-through. In this work we pre- 


\section{Coloproctology y}

sented an innovative TEM-endoscopy assisted approach to treat postoperative complication of modified Duhamel operation. This is a mini-invasive approach that allows to perform a new colorectal anastomosis by a transanal access. The postoperative progress was regular without any surgical complication. No stenosis recurrence has been observed at 1-year follow-up. This is an original contribution because no similar procedures were described in literatures. In our opinion; a wide experience is needed to perform this kind of surgery.

We describe a successful technique for the management of a technically difficult post-operative colorectal complete stenosis. Transanal approach by TEM-endoscopy assisted represents a safe and feasible model of minimally invasive surgical technique.

\section{REFERENCES}

1. Auyang ED, Santos BF, Enter DH, Hungness ES, Soper NJ. Natural orifice translumenal endoscopic surgery (NOTES(R)): a technical review. Surg Endosc 2011;25:3135-48.

2. D’Ambrosio G, Paganini AM, Guerrieri M, Barchetti L, Lezoche G, Fabiani B, et al. Minimally invasive treatment of rectovaginal fistula. Surg Endosc 2012;26:546-50.

3. Morks AN, Ploeg RJ, Sijbrand Hofker H, Wiggers T, Havenga K. Late anastomotic leakage in colorectal surgery: a significant problem. Colorectal Dis 2013;15:e271-5.

4. Fregonese D, Di Falco G, Di Toma F. Balloon dilatation of anastomotic intestinal stenoses: long-term results. Endoscopy 1990;22: 249-53.

5. Truong S, Willis S, Schumpelick V. Endoscopic therapy of benign anastomotic strictures of the colorectum by electroincision and balloon dilatation. Endoscopy 1997;29:845-9. 